Research Paper

\title{
Prognostic value of stem cell markers in esophageal and esophagogastric junction cancer: a meta-analysis
}

\author{
Elisabetta Trevellin ${ }^{1 凶}$, Giovanni Pirozzolo ${ }^{2}$, Matteo Fassan ${ }^{3}$, Roberto Vettor ${ }^{1}$ \\ 1. Department of Medicine, Endocrine-Metabolic Laboratory, University of Padua, 35128 Padua, Italy \\ 2. Emergency General Surgery, Ospedale dell'Angelo - ULSS 3 Serenissima, 30174 Venice, Italy \\ 3. Department of Medicine, Surgical Pathology Unit, University of Padua, 35128 Padua, Italy \\ $\square$ Corresponding author: Elisabetta Trevellin MSc, PhD. Department of Medicine - DIMED - Endocrine and Metabolic Lab, University of Padova, via Ospedale, \\ 105, 35128 Padova - Italy. phone: +39 0498213032; fax: +39 0498218555; e-mail: elisabetta.trevellin@unipd.it
}

(c) The author(s). This is an open access article distributed under the terms of the Creative Commons Attribution License (https://creativecommons.org/licenses/by/4.0/). See http://ivyspring.com/terms for full terms and conditions.

Received: 2019.01.31; Accepted: 2020.04.14; Published: 2020.04.27

\begin{abstract}
Background: Esophageal cancer is an aggressive tumor, with poor prognosis and low survival rates. Although diagnosis and treatment have improved considerably, more efficient prognostic factors are urgently needed to prevent postoperative recurrence and metastasis. Cancer stem cells are key players in tumor progression and several studies have investigated the association between the expression of stemness genes and clinical outcome. However, the prognostic value of stemness markers in esophageal cancer remains controversial. We identified six factors involved in angiogenesis, anti-apoptosis and self-renewal that have been associated to poor prognosis in other types of cancer. We conducted a review of the literature and a meta-analysis to assess their potential prognostic role in this malignancy.

Material and Methods: The database of PMC, PubMed, Web of Science, Embase and The Cochrane Library were searched to investigate the association between CD34, CD133, Nucleostemin, OCT-4, NANOG and CD90, and the survival of patients affected by esophageal squamous cell carcinoma or esophageal adenocarcinoma. Among the 615 eligible studies, a total of 19 articles (including 1586 patients) met the inclusion criteria for the meta-analysis, and the pooled hazard ratio and $95 \%$ confidence intervals were calculated.

Results: Data showed that high expression of CD34 (HR 2.10; 95\%Cl 1.41-3.14; $12=56 \%$; $\mathrm{p}=0.0003$ ), CD133 (HR 1.91; 95\%Cl 1.15-3.19; |2=55\%; $p=0.01$ ) and Nucleostemin (HR 2.97; 95\%Cl 1.11-7.98; |2=0\%; $p=0.03$ ) were associated with poor prognosis in patients affected by esophageal cancer. The expression of NANOG and OCT-4 showed no significant association with survival of patients, whereas no study involving CD90 was included in this meta-analysis. Conclusion: CD34, CD133 and Nucleostemin might represent useful prognostic markers in patients affected by esophageal cancer.
\end{abstract}

Key words: Esophageal cancer; Cancer stem cells; CD34; CD133; Nucleostemin; Neoadjuvant therapy; Prognosis

\section{Introduction}

Esophageal cancer is one of the most unknown and deadliest cancers worldwide, characterized by an aggressive nature and poor survival rate [1]. Esophageal cancer typically occurs in two histologic forms: squamous cell carcinoma (SCC) is the predominant form, arising from the stratified squamous epithelial lining of the organ, whereas esophageal adenocarcinoma (EAC) affects columnar glandular cells that replace the squamous epithelium [2]. Although current treatment options (including surgery, radiation and chemotherapy) are constantly improving, the overall survival remains poor and stronger prognostic factors are needed. Cancer stem cells (CSCs) may play an important role in the progression and prognosis of esophageal cancers, by expressing factors involved in angiogenesis, neoangiogenesis, anti-apoptosis and self-renewal [3]. Some studies showed that a higher expression of the stemness markers CD34, CD90, Nucleostemin, CD133, OCT-4 and NANOG in tumoral tissue of 
patients correlated with poor prognosis in different types of tumors. In particular, CD34 is reduced in breast cancer [4] and prostate cancer [5] after neoadjuvant treatment and is usually related to VEGF expression, which negatively correlates to the response to neoadjuvant therapy in esophageal cancer tissue [6]. CD133 has been shown to be associated with worse prognosis in NSCLC [7], ovarian cancer [8], hepatocellular carcinoma [9], breast cancer [10] and colorectal cancer [11], however its role in esophageal cancer still needs to be investigated. CD90 is upregulated in cancer-associated fibroblasts and correlated with recurrence in hepatocellular carcinoma [12] and with survival in neuroblastoma [13]. Nucleostemin is upregulated in recurrent esophageal carcinoma [14], in advanced malignant phenotype of oral squamous cell carcinoma [15] and in human breast cancer cells resistant to chemotherapy [16]. OCT-4 is essential for anti-apoptosis in chemoresistant cell lines [17] and is increased in tumor treated with neoadjuvant therapy [18]. NANOG is an early-differentiation marker that has been associated with worse prognosis in tongue squamous cell carcinoma [19], ovarian serous carcinoma [20] and breast cancer [21]. The analysis of these markers in esophageal cancer tissue may potentially lead to better prognosis as well as play a part in assessing tumor response to therapy; however, their prognostic role is still not clear to date.

Antibody-based agents like ipilimumab, pembrolizumab, and nivolumab are currently used to block CTLA-4 and PD-1 binding to PDL-1 respectively, effectively enhancing endogenous immune responses and antitumor activity. Esophageal cancer is also being explored in terms of immune checkpoint inhibition trials and early results seem promising in esophageal SCC and gastric adenocarcinoma [22]. Recent studies implicated CSCs to play a role in tumor chemoradio-resistance and response to CRT so CSC markers might be used to select patients who would not benefit from conventional CRT but would need other therapy such as immunotherapy [23]. On the other hand, as observed in glioma, CSCs (CD133-positive cells) are able to repair DNA damage more efficiently and rapidly than CD133 negative cells and this might decrease the tumor mutational load and, by consequence, tumor immunogenicity [24]. Therefore, the use of CSC markers to predict the need and the effect of immunotherapy is still under debate. In this study, we collected the data available in literature and conducted a meta-analysis to clarify the prognostic value for each marker in esophageal cancer.

\section{Materials and Methods}

\section{Literature search and eligibility Criteria}

This review was registered with the International Prospective Register for Systematic Reviews (PROSPERO) platform under the number: CRD42017058771. The database of PMC, PubMed, Web of Science, Embase and The Cochrane Library were searched in March 2017 and the search strategy was the following: (CD34 OR CD90 OR Nucleostemin OR CD133 OR "OCT4" OR "OCT-4" OR NANOG) AND (esophageal OR oesophageal OR esophagus OR oesophagus OR esophagogastric junction) AND (cancer OR tumor OR carcinoma OR adenocarcinoma OR neoplasm). The inclusion criteria were: 1) the diagnosis of SCC or EAC was based on pathological examination; 2) the expression of CD34 or CD90 or Nucleostemin or CD133 or OCT-4 or NANOG with OS/DSS/DFS was reported; 3) HRs and 95\% CIs were provided in text or sufficient data was provided for the calculation of HRs and 95\% CIs; 4) articles published as original research. To avoid duplicate data, we selected only the more recent or complete article when multiple reports described the same population. The exclusion criteria were: 1) reviews, meeting abstracts, letters; 2) non-primitive tumors; 3 ) case-reports; 4) animal or in vitro studies; 5) sample size < 10 patients. Two researchers (E.T. and M.S.) independently selected studies that matched the inclusion criteria. Any discordance was resolved by discussion.

\section{Data extraction}

Two researchers (E.T. and M.S.) independently extracted the following data: author, year of publication, study center and country, sample size, demographic data, clinicopathological parameters, cut-off value of CD34 or CD90 or Nucleostemin or CD133 or OCT-4 or NANOG expression, survival data, follow up duration, tumor location, neoadjuvant therapy characteristics, methodological data, overall survival (OS) hazard ratio (HR), disease free survival (DFS) HR, progression free survival (PFS) HR. HR were extracted both from multivariate and univariate analysis, preferring data from multivariate analysis when available. When HR was not declared it was extracted from Kaplan-Meyer curves following the method described by Parmar [25].

\section{Quality assessment}

Two researchers (E.T. and M.S.) independently assessed the quality of included studies by the Newcastle-Ottawa Scale (NOS) for assessing the quality of non-randomised studies in meta-analyses. 
Studies with NOS scores of less than 5 were not included in the meta-analysis.

\section{Statistical analysis}

Extracted data were analysed using RevMan 5.3 analysis software. Generic inverse variance was used to pool hazard ratios. Fixed-effect model and random-effect model were used depending on heterogeneity. Heterogeneity, assessed using $\mathrm{I}^{2}$ statistic, was considered relevant when $>30 \%$ [26]. Funnel plot asymmetry was estimated by visual inspection to assess publication bias of the included studies for each stemness marker [26]. Statistical significance was considered relevant when $p<0.05$.

\section{Results}

\section{Study Characteristics}

The selection process of the eligible studies is presented in Figure 1. A total of 19 articles [14, 27-44] including 1586 patients met the inclusion criteria for the meta-analysis. The basic characteristics of each eligible study and the NOS scores of each study are summarized in Table 1. All articles were published between 1996 and 2017, most of the studies were conducted in Asia $(n=15)$ and the remaining were conducted in Europe $(n=4)$. Seven studies involved the analysis of the marker CD34, five studies the analysis of CD133, four studies the analysis of OCT-4, three studies the analysis of NANOG and two studies the analysis of Nucleostemin, whereas none of the selected studies involved the analysis of CD90. The majority of the studies were conducted on patients affected by SCC $(n=18)$ and, among them, one study [35] also involved patients affected by EAC. One study [27] only involved patients with EAC. The detection method was immunohistochemistry (IHC) for all the studies except for one [30], in which mRNA in situ hybridization (ISH) was used. The cut-off values were expressed as percentage of positive cells, number of positive cells or as a score determined on the intensity of immunohistochemical expression. Eleven studies explored the prognostic value of a marker in overall survival (OS), three studies in cumulative survival (CS), two studies in disease-free survival (DFS), two studies in the onset of lymphatic metastasis, one study in relapse-free survival (RFS), one study in cause-specific survival (CSS) and one in esophageal cancer-specific survival (ECSS). The information regarding name (clones), manufacturer and (if available) dilution of the antibodies used to perform the staining in each study are summarized in Table 2.

Table 1. Characteristics of included studies. SCC: squamous cell carcinoma; EAC: esophageal adenocarcinoma; IHC: immunohistochemistry; ISH: in situ hybridization; RFS: relapse-free survival; DSF: disease-free survival; OS: overall survival; CSS cause-specific survival; ECSS: esophageal cancer-specific survival

\begin{tabular}{|c|c|c|c|c|c|c|c|c|c|c|c|}
\hline \multirow[t]{2}{*}{ AUTHOR } & \multirow[t]{2}{*}{ YEAR } & \multirow[t]{2}{*}{$\begin{array}{l}\text { STEM CELL } \\
\text { MARKER }\end{array}$} & \multirow[t]{2}{*}{ COUNTRY } & \multirow[t]{2}{*}{ ETHNICITY } & \multicolumn{2}{|c|}{$\begin{array}{l}\text { TUMOR } \\
\text { TYPE }\end{array}$} & \multirow[t]{2}{*}{$\begin{array}{l}\text { DETECTION } \\
\text { METHOD }\end{array}$} & \multirow[t]{2}{*}{ CUT-OFF VALUE } & \multirow[t]{2}{*}{ OUTCOME } & \multirow{2}{*}{$\begin{array}{l}\text { HAZARD RATIO } \\
\text { (REPORTED/ } \\
\text { ESTIMATED) }\end{array}$} & \multirow[t]{2}{*}{$\begin{array}{l}\text { NOS } \\
\text { SCORE }\end{array}$} \\
\hline & & & & & SCC & EAC & & & & & \\
\hline Perry et al. & 2015 & CD34 & UK & Caucasian & 0 & 43 & IHC & $>0$ & RFS & $\mathrm{E}$ & 6 \\
\hline Lu et al. & 2015 & CD133 & China & Asian & 154 & 0 & IHC & Score $\geq 2(0-3)$ & DFS OS & $\mathrm{R}$ & 5 \\
\hline $\begin{array}{l}\text { Shimada et } \\
\text { al. }\end{array}$ & 2012 & $\begin{array}{l}\text { NANOG and } \\
\text { OCT-4 }\end{array}$ & Japan & Asian & 81 & 0 & IHC & $\begin{array}{l}\text { NANOG: Score }>2 \\
(0-5) ; \text { OCT-4 Score >3 } \\
(0-5)\end{array}$ & CSS & $\mathrm{E}$ & 5 \\
\hline Zhang et al. & 2010 & Nucleostemin & China & Asian & 62 & 0 & ISH & $>0$ & $\begin{array}{l}\text { Lymphatic } \\
\text { Metastasis }\end{array}$ & E & 6 \\
\hline He et al. & 2012 & OCT-4 & China & Asian & 153 & 0 & IHC & Score $>3(0-9)$ & OS & $\mathrm{R}$ & 6 \\
\hline $\begin{array}{l}\text { Okamoto et } \\
\text { al. }\end{array}$ & 2016 & CD133 & Japan & Asian & 47 & 0 & IHC & $>80 \%$ & ECSS & $\mathrm{R}$ & 6 \\
\hline Yang et al. & 2017 & OCT-4 & Korea & Asian & 127 & 0 & IHC & Score $\geq 2(0-3)$ & DFS OS & $\mathrm{R}$ & 6 \\
\hline $\begin{array}{l}\text { Nakajima } \\
\text { et al. }\end{array}$ & 2012 & $\begin{array}{l}\text { Nucleostemin } \\
\text { and CD133 }\end{array}$ & Japan & Asian & 51 & 0 & IHC & $\begin{array}{l}\text { Nucleostemin }>80 \% \text {; } \\
\text { CD133 >0 }\end{array}$ & OS & $\mathrm{R}$ & 7 \\
\hline Li et al. & 2012 & OCT-4 & China & Asian & 50 & 0 & IHC & Score $\geq 3(0-4)$ & OS & $\mathrm{R}$ & 6 \\
\hline $\begin{array}{l}\text { Goscinski } \\
\text { et al. }\end{array}$ & 2013 & CD34 & Norway & Caucasian & 24 & 28 & IHC & $>25 \%$ & CS & $\mathrm{E}$ & 5 \\
\hline Elpek et al. & 2001 & CD34 & Turkey & Caucasian & 53 & 0 & IHC & $\geq 92$ & OS & E & 6 \\
\hline $\begin{array}{l}\text { Igarashi et } \\
\text { al. }\end{array}$ & 1998 & CD34 & Japan & Asian & 83 & 0 & IHC & $>116$ & OS & E & 6 \\
\hline Hang et al. & 2012 & CD133 & China & Asian & 110 & 0 & IHC & $>1 \%$ & OS & E & 7 \\
\hline $\begin{array}{l}\text { Hwang et } \\
\text { al. }\end{array}$ & 2014 & NANOG & Taiwan & Asian & 41 & 0 & IHC & $>0$ & CS & $\mathrm{E}$ & 5 \\
\hline $\begin{array}{l}\text { Okamoto et } \\
\text { al. }\end{array}$ & 2013 & CD133 & Japan & Asian & 86 & 0 & IHC & $>1 \%$ & OS & $\mathrm{R}$ & 7 \\
\hline $\begin{array}{l}\text { Kitadai et } \\
\text { al. }\end{array}$ & 1998 & CD34 & Japan & Asian & 71 & 0 & IHC & $>43$ & OS & E & 5 \\
\hline Sun et al. & 2015 & NANOG & China & Asian & 149 & 0 & IHC & $>8(0-12)$ & CS & E & 6 \\
\hline $\begin{array}{l}\text { Tanigawa } \\
\text { et al. }\end{array}$ & 1997 & CD34 & Japan & Asian & 43 & 0 & IHC & $\geq 145$ & OS & $\mathrm{R}$ & 6 \\
\hline Sarbia et al. & 1996 & CD34 & Germany & Caucasian & 130 & 0 & IHC & $>63$ & $\begin{array}{l}\text { Lymphatic } \\
\text { Metastasis }\end{array}$ & E & 6 \\
\hline
\end{tabular}



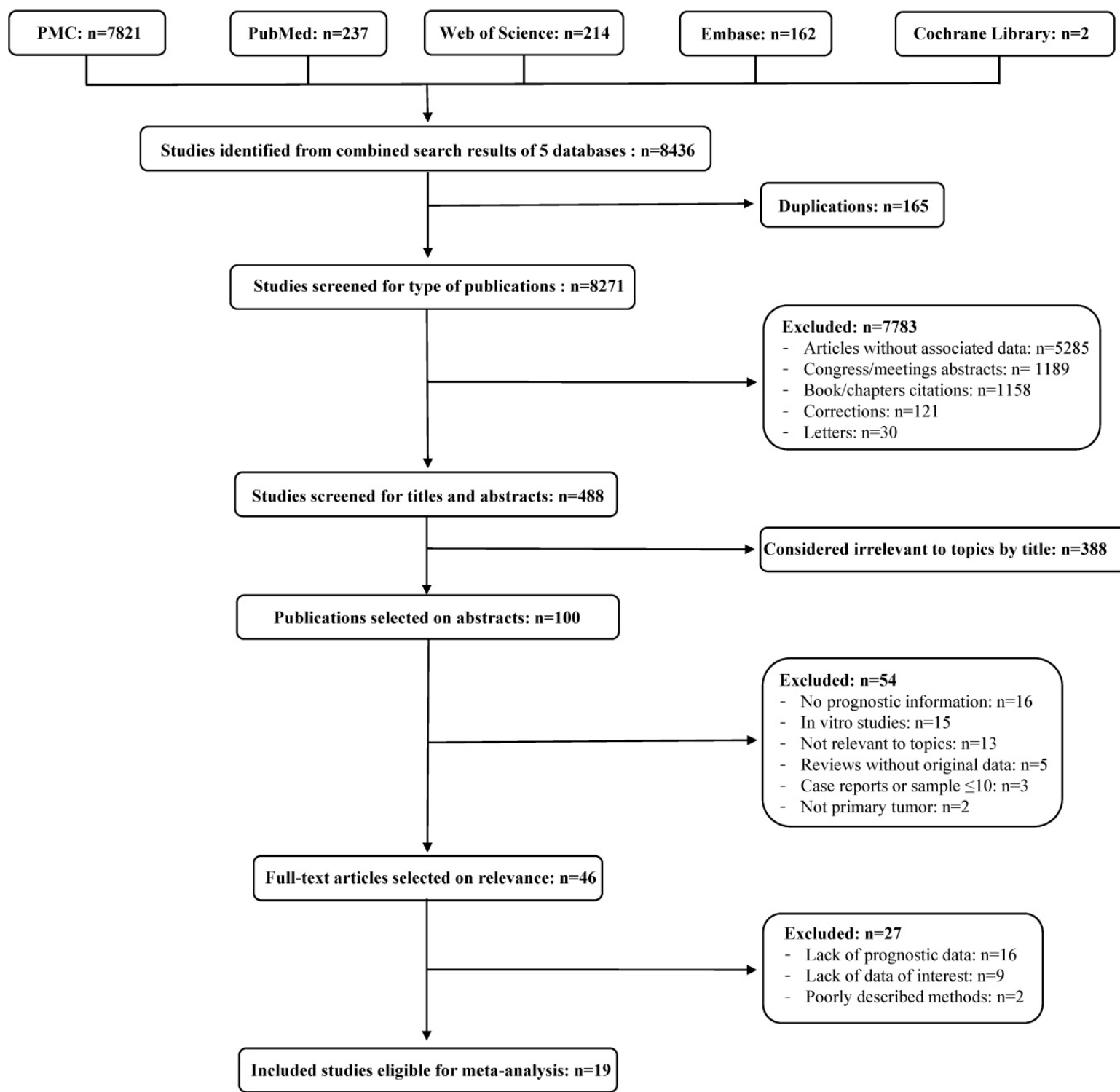

Figure 1. Flow diagram of the literature review process. Identified, included and excluded studies are shown in the different steps of the inspection.

Table 2. Characteristics of antibodies used for staining procedures in the included studies. NA: not available

\begin{tabular}{|c|c|c|c|c|}
\hline AUTHOR & YEAR & STEM CELL MARKER & ANTIBODY & DILUTION \\
\hline Perry et al. & 2015 & CD34 & Anti-CD34 (MCAP547, Serotec, Oxford, UK) & $1: 500$ \\
\hline Lu et al. & 2015 & CD133 & Anti-CD133 (Cloud-Clone Corp, Houston, Tex) & NA \\
\hline Shimada et al. & 2012 & NANOG and OCT-4 & $\begin{array}{l}\text { Anti-NANOG (IHC-00205; Bethyl Laboratories, Montogomery, TX, USA) Anti-OCT4 } \\
\text { (ab19857; Abcam) }\end{array}$ & NA \\
\hline Zhang et al. & 2009 & Nucleostemin & Anti-human nucleostemin (R\&D Systems, Minneapolis, MN, USA) & NA \\
\hline He et al. & 2012 & OCT-4 & Anti-OCT4 (AF1759, R\&D Systems, Minneapolis, MN, USA) & $1: 40$ \\
\hline Okamoto et al. & 2016 & CD133 & Anti-CD133 (PROM-1, Abnova Corporation, Taipei, Taiwan) & $1: 250$ \\
\hline Yang et al. & 2017 & OCT-4 & Anti-Oct4 (Millipore, USA) & 1:100 \\
\hline Nakajima et al. & 2012 & Nucleostemin and CD133 & $\begin{array}{l}\text { Anti-nucleostemin (A300-600A; Bethyl Laboratories, Montgomery, TX, USA) Anti-CD133 } \\
\text { (130-092-395; Miltenyi Biotec, Auburn, CA, USA) }\end{array}$ & 1:100 1:250 \\
\hline Li et al. & 2012 & OCT-4 & Anti-human OCT4 (Santa Cruz Biotechnology, Inc. Santa Cruz, CA, USA) & NA \\
\hline Goscinski et al. & 2013 & CD34 & Anti-CD34 (QBend-10, Monosan, The Netherlands) & $1: 1000$ \\
\hline Elpek et al. & 2001 & CD34 & Anti-CD34 (Qbend-10, Dako, Glostrup, Denmark) & $1: 50$ \\
\hline Igarashi et al. & 1998 & CD34 & Anti-CD34 (Biogenesis Inc., Poole, UK) & 1:500 \\
\hline Hang et al. & 2012 & CD133 & Anti-CD133 (C24B9, Cell Signaling Technologies, Danvers, MA, USA) & $1: 100$ \\
\hline Hwang et al. & 2014 & NANOG & Anti-NANOG (Epitomics, Burlingame CA, USA) & NA \\
\hline Okamoto et al. & 2013 & CD133 & Anti-CD133 (AC133, Miltenyi Biotec, Auburn, CA, USA) & $1: 10$ \\
\hline Kitadai et al. & 1998 & CD34 & Anti-CD34 (Nichirei, Tokyo, Japan) & $1: 300$ \\
\hline Sun et al. & 2015 & NANOG & Anti-NANOG (Cell Signaling Technologies, Danvers, MA, USA) & $1: 200$ \\
\hline Tanigawa et al. & 1997 & CD34 & Anti-CD34 (QBend-10, Novocastra Labo, Newcastle, UK) & $1: 25$ \\
\hline Sarbia et al. & 1996 & CD34 & Anti-CD34 (QBend-10, Serotec, Oxford, UK) & NA \\
\hline
\end{tabular}


Table 3. Characteristics of patients enrolled in the included studies. EG]: esophagogastric junction; NA: not available; CT: chemotherapy; RT: radiotherapy.

\begin{tabular}{|c|c|c|c|c|c|c|c|c|c|c|c|c|c|c|c|}
\hline \multirow[t]{2}{*}{ AUTHOR } & \multicolumn{2}{|c|}{ SAMPLE SIZE } & \multicolumn{2}{|c|}{ SEX } & \multirow[t]{2}{*}{ AGE } & \multirow[t]{2}{*}{$\begin{array}{l}\text { FOLLOW UP } \\
\text { (MONTHS) }\end{array}$} & \multicolumn{4}{|c|}{ TUMOR STAGE } & \multicolumn{3}{|c|}{ TUMOR LOCATION } & \multicolumn{2}{|c|}{$\begin{array}{l}\text { NEOADJUVANT } \\
\text { THERAPY }\end{array}$} \\
\hline & $\begin{array}{l}\text { Total } \\
\text { Patients }\end{array}$ & $\begin{array}{l}\text { Actual } \\
\text { Patients }\end{array}$ & $\mathbf{M}$ & $\mathbf{F}$ & & & I & II & III & IV & $\begin{array}{l}\text { EGJ/ } \\
\text { lower }\end{array}$ & thoracic/middle & cervical/upper & CT & RT \\
\hline Perry et al. & 43 & 43 & 32 & 11 & 68,2 & $43(3-122)$ & NA & NA & NA & NA & 23 & 20 & & 0 & 0 \\
\hline Lu et al. & 154 & 154 & 119 & 35 & $\begin{array}{l}\leq 60=76 ; \\
>60=78\end{array}$ & 108 & 12 & 83 & 59 & 0 & NA & NA & NA & 0 & 0 \\
\hline $\begin{array}{l}\text { Shimada et } \\
\text { al. }\end{array}$ & 114 & 81 & 72 & 9 & 64,5 & 40 & 11 & 38 & 36 & 8 & NA & NA & NA & 0 & 0 \\
\hline Zhang et al. & 62 & 62 & 36 & 26 & $\begin{array}{l}<60=29 ; \\
>60=33\end{array}$ & not mentioned & 7 & 28 & 26 & 1 & NA & NA & NA & 0 & 0 \\
\hline He et al. & 153 & 153 & 93 & 60 & 56,4 & $124(118-155)$ & 5 & 39 & 100 & 9 & 35 & 101 & 14 & 0 & 0 \\
\hline $\begin{array}{l}\text { Okamoto et } \\
\text { al. }\end{array}$ & 47 & 47 & 40 & 7 & & $42(6-82)$ & 12 & & 35 & & 14 & 27 & 6 & 47 & 0 \\
\hline Yang et al. & 147 & 127 & 120 & 7 & $\begin{array}{l}<65=34 \\
>65=93\end{array}$ & 120 & 15 & 112 & & & NA & NA & NA & 0 & 0 \\
\hline $\begin{array}{l}\text { Nakajima et } \\
\text { al. }\end{array}$ & 54 & 51 & 47 & 7 & $62(36-74)$ & until relapse & 2 & 11 & 33 & 8 & NA & NA & NA & 51 & 0 \\
\hline Li et al. & 50 & 50 & 37 & 13 & $62(47-72)$ & 80 & 14 & 31 & 5 & & NA & NA & NA & 0 & 0 \\
\hline $\begin{array}{l}\text { Goscinski et } \\
\text { al. }\end{array}$ & 52 & 52 & 42 & 10 & $38-87$ & 60 & NA & NA & NA & NA & NA & NA & NA & 0 & 0 \\
\hline Elpek et al. & 53 & 53 & 30 & 23 & $42(32-55)$ & $24(6-60)$ & 26 & & 27 & & 22 & 19 & 12 & 0 & 0 \\
\hline $\begin{array}{l}\text { Igarashi et } \\
\text { al. }\end{array}$ & 93 & 83 & 84 & 9 & $64,3(44-83)$ & $28-36$ & 44 & & 59 & & 33 & 47 & 13 & 0 & 0 \\
\hline Hang et al. & 110 & 110 & 61 & 49 & $57(38-81)$ & 24 & 11 & 65 & 34 & & 14 & 72 & 24 & 0 & 0 \\
\hline Hwang et al. & 41 & 41 & 39 & 2 & $54(37-78)$ & $13(0,3-57,4)$ & & 7 & 23 & 11 & NA & NA & NA & 41 & 41 \\
\hline $\begin{array}{l}\text { Okamoto et } \\
\text { al. }\end{array}$ & 86 & 86 & 73 & 13 & $64(37-81)$ & until death & 20 & 28 & 3 & 5 & 41 & 35 & 10 & 0 & 0 \\
\hline Kitadai et al. & 119 & 71 & 107 & 12 & $63,5(39-86)$ & 120 & 67 & & 52 & & NA & NA & NA & 81 & 81 \\
\hline Sun et al. & 149 & 149 & 112 & 37 & 54,0 & until death & 4 & 82 & 54 & 9 & NA & NA & NA & 0 & 0 \\
\hline $\begin{array}{l}\text { Tanigawa et } \\
\text { al. }\end{array}$ & 43 & 43 & 28 & 15 & $65(46-81)$ & 24 & 18 & 9 & 6 & 10 & 15 & 20 & 8 & 2 & 6 \\
\hline Sarbia et al. & 150 & 130 & 121 & 29 & $58(35-82)$ & 24-192 & 72 & & 78 & & NA & NA & NA & 0 & 0 \\
\hline
\end{tabular}

\section{Patient characteristics}

The characteristics of patients involved in the 19 studies included in the meta-analysis are summarized in Table 3. A total of 1586 patients (1253 male and 360 female) were analyzed, the median age ranged from 42 to 68.2 and the months of follow up ranged from 0.3 to 192. In 17 studies, the stages of tumor (from I to IV) were reported and in 8 studies the location of tumor (lower, middle or upper esophagus) was specified. In 6 studies the patients underwent neoadjuvant therapy (308 patients underwent chemotherapy and 148 underwent radiotherapy) before surgery.

\section{Prognostic value of CD34 in esophageal cancer}

We conducted a meta-analysis of the studies $[35-37,41,44]$ that reported the analysis of the marker CD34. These studies involved 475 patients, most of them with SCC (404) and the remaining 71 with EAC. Tumor was localized mainly in the middle or lower portion of esophagus and most of the patients (396) did not undergo neoadjuvant therapy before surgery (and thus sampling). Due to the presence of only one article involving patients affected by EAC, we decided to exclude those data from the meta-analysis to avoid any bias caused by the different type of tumor. Our study showed a significant association between high CD34 expression in tumoral tissue and a poorer prognosis in patients affected by esophageal cancer (HR 2.10; 95\%CI 1.41-3.14; I2 56\%; p=0.0003) (Figure 2A).

\section{Prognostic value of CD133 in esophageal cancer}

Studies involving CD133 [14, 28, 32, 38, 40] were analyzed. These studies involved 448 patients with SCC. Tumor was localized mainly in the middle portion of esophagus and most of the patients (350) did not undergo neoadjuvant therapy before surgery. The meta-analysis showed a significant association between high expression of CD133 in tumoral tissue and poor prognosis in patients affected by esophageal cancer (HR 1.91; 95\%CI 1.15-3.19; I $\mathrm{I}^{2}$ 55\%; $\mathrm{p}=0.01$ ) (Figure 2B).

\section{Prognostic value of Nucleostemin in esophageal cancer}

Two studies [14, 30] involved the analysis of Nucleostemin and included a total of 113 patients with SCC, of which 51 received neoadjuvant therapy before surgery. The meta-analysis that we carried out showed a significant association between high expression of Nucleostemin in tumoral tissue and poor prognosis in patients affected by esophageal cancer (HR 2.97; 95\%CI 1.11-7.98; $\mathrm{I}^{2} 0 \%$; $\mathrm{p}=0.03$ ) (Figure 2C). 
A

\begin{tabular}{|c|c|c|c|c|c|c|c|c|}
\hline $\begin{array}{l}\text { CD34 } \\
\text { Study or Subgroup }\end{array}$ & log[Hazard Ratio] & SE & Weight & $\begin{array}{l}\text { Hazard Ratio } \\
\text { IV, Random, } 95 \% \mathrm{CI}\end{array}$ & & $\begin{array}{r}\text { Hazard } \\
\text { IV, Randor }\end{array}$ & $\begin{array}{l}\text { d Ratio } \\
\mathrm{m}, 95 \% \mathrm{Cl}\end{array}$ & \\
\hline Elpek 2001 & 1.16 & 0.3553 & $16.6 \%$ & $3.19[1.59,6.40]$ & & & $\Longrightarrow$ & \\
\hline Goscinski 2013 & 1.2119 & 0.4884 & $11.5 \%$ & $3.36[1.29,8.75]$ & & & & \\
\hline Igarashi 1998 & 0.6206 & 0.2966 & $19.5 \%$ & $1.86[1.04,3.33]$ & & & $\rightleftarrows$ & \\
\hline Kitadai 1998 & 0.6627 & 0.2803 & $20.4 \%$ & $1.94[1.12,3.36]$ & & & $\longrightarrow$ & \\
\hline Sarbia 1996 & 0.2231 & 0.1676 & $27.0 \%$ & $1.25[0.90,1.74]$ & & & $=$ & \\
\hline Tanigawa 1997 & 1.911 & 0.838 & $5.1 \%$ & $6.76[1.31,34.93]$ & & & & \\
\hline Total $(95 \% \mathrm{Cl})$ & & & $100.0 \%$ & $2.10[1.41,3.14]$ & & & & \\
\hline \multicolumn{5}{|c|}{$\begin{array}{l}\text { Heterogeneity. } \mathrm{Tau}^{2}=0.13 ; \mathrm{Chi}^{2}=11.44, \mathrm{df}=5(P=0.04) ;\left.\right|^{2}=56 \% \\
\text { Test for overall effect: } Z=3.64(P=0.0003)\end{array}$} & $\lcm{0.01}$ & $\begin{array}{c}0.1 \\
\text { Favours BETTER prognosis }\end{array}$ & $\begin{array}{c}10 \\
10 \\
\text { Favours WORSE prognosis }\end{array}$ & $\overrightarrow{100}$ \\
\hline
\end{tabular}

B

\begin{tabular}{|c|c|c|c|c|c|c|}
\hline \multicolumn{4}{|l|}{ CD133 } & $\begin{array}{l}\text { Hazard Ratio } \\
\text { IV, Random, } 95 \% \mathrm{CI}\end{array}$ & \multicolumn{2}{|c|}{$\begin{array}{c}\text { Hazard Ratio } \\
\text { IV, Random, } 95 \% \mathrm{CI}\end{array}$} \\
\hline Hang 2012 & 0.8629 & 0.2759 & $28.8 \%$ & $2.37[1.38,4.07]$ & & $\longrightarrow$ \\
\hline Lu 2015 & 0.0733 & 0.2341 & $31.7 \%$ & $1.08[0.68,1.70]$ & - & - \\
\hline Nakajima 2012 & 1.4816 & 1.061 & $5.3 \%$ & $4.40[0.55,35.20]$ & & \\
\hline Okamoto 2013 & 0.5539 & 0.3142 & $26.3 \%$ & $1.74[0.94,3.22]$ & & $=$ \\
\hline Okamoto 2016 & 1.9137 & 0.8347 & $8.0 \%$ & $6.78[1.32,34.80]$ & & \\
\hline Total $(95 \% \mathrm{Cl})$ & & & $100.0 \%$ & $1.91[1.15,3.19]$ & & \\
\hline \multicolumn{5}{|c|}{$\begin{array}{l}\text { Heterogeneity. } \text { Tau }^{2}=0.16 ; \mathrm{Chi}^{2}=8.80, \mathrm{df}=4(\mathrm{P}=0.07) ; \mathrm{I}^{2}=55 \% \\
\text { Test for overall effect: } \mathrm{Z}=2.48(\mathrm{P}=0.01)\end{array}$} & $\begin{array}{|lc|}0.01 & 0.1 \\
& \text { Favours BETTER prognosis }\end{array}$ & $\begin{array}{c}10 \\
\text { Favours WORSE prognosis }\end{array}$ \\
\hline
\end{tabular}

C

\begin{tabular}{|c|c|c|c|c|c|c|c|c|}
\hline $\begin{array}{l}\text { Nucleostemin } \\
\text { Study or Subgroup }\end{array}$ & log[Hazard Ratio] & SE & Weight & $\begin{array}{l}\text { Hazard Ratio } \\
\text { IV, Fixed, } 95 \% \mathrm{CI}\end{array}$ & & $\begin{array}{r}\text { Hazard } \\
\text { IV, Fixed, }\end{array}$ & $\begin{array}{l}\text { Ratio } \\
95 \% \mathrm{Cl}\end{array}$ & \\
\hline $\begin{array}{l}\text { Nakajima } 2012 \\
\text { Zhang } 2009\end{array}$ & $\begin{array}{l}1.4563 \\
0.9933\end{array}$ & $\begin{array}{r}1.107 \\
0.5662\end{array}$ & $\begin{array}{l}20.7 \% \\
79.3 \%\end{array}$ & $\begin{array}{r}4.29[0.49,37.56] \\
2.70[0.89,8.19]\end{array}$ & & & & \\
\hline Total $(95 \% \mathrm{Cl})$ & & & $100.0 \%$ & $2.97[1.11,7.98]$ & & & & \\
\hline \multicolumn{4}{|c|}{$\begin{array}{l}\text { Heterogeneity. Chi } \mathrm{I}^{2}=0.14, \mathrm{df}=1(P=0.71) ; \mathrm{I}^{2}=0 \% \\
\text { Test for overall effect: } Z=2.16(P=0.03)\end{array}$} & & 0.01 & $\begin{array}{c}0.1 \\
\text { Favours BETTER prognosis }\end{array}$ & $\begin{array}{c}10 \\
10 \\
\text { Favours WORSE prognosis }\end{array}$ & $\overrightarrow{100}$ \\
\hline
\end{tabular}

D

\begin{tabular}{|c|c|c|c|c|c|c|c|c|}
\hline $\begin{array}{l}\text { OCT-4 } \\
\text { Study or Subgroup }\end{array}$ & log[Hazard Ratio] & SE & Weight & $\begin{array}{l}\text { Hazard Ratio } \\
\text { IV, Random, } 95 \% \mathrm{CI}\end{array}$ & & $\begin{array}{r}\text { Hazard } \\
\text { IV, Random }\end{array}$ & $\begin{array}{l}\text { Ratio } \\
\text { n, } 95 \% \mathrm{Cl}\end{array}$ & \\
\hline He 2012 & 0.9746 & 0.2803 & $25.5 \%$ & $2.65[1.53,4.59]$ & & & $\longrightarrow$ & \\
\hline Li 2012 & 1.3002 & 0.4268 & $20.5 \%$ & $3.67[1.59,8.47]$ & & & & \\
\hline Shimada 2012 & 0.1989 & 0.2415 & $26.8 \%$ & $1.22[0.76,1.96]$ & & & & \\
\hline Yang 2017 & -0.2107 & 0.2261 & $27.3 \%$ & $0.81[0.52,1.26]$ & & $\rightarrow+$ & & \\
\hline Total $(95 \% \mathrm{Cl})$ & & & $100.0 \%$ & $1.67[0.88,3.17]$ & & & & \\
\hline \multicolumn{5}{|c|}{$\begin{array}{l}\text { Heterogeneity. } \text { Tau }^{2}=0.34 ; \mathrm{Chi}^{2}=16.46 \text {, df }=3(P=0.0009) ; \mathrm{I}^{2}=82 \% \\
\text { Test for overall effect: } Z=1.55(P=0.12)\end{array}$} & 0.01 & $\begin{array}{cc}0.1 & 1 \\
\text { Favours } B E T T E R & \text { prognosis }\end{array}$ & $\begin{array}{c}10 \\
\text { Favours WORSE prognosis }\end{array}$ & 100 \\
\hline
\end{tabular}

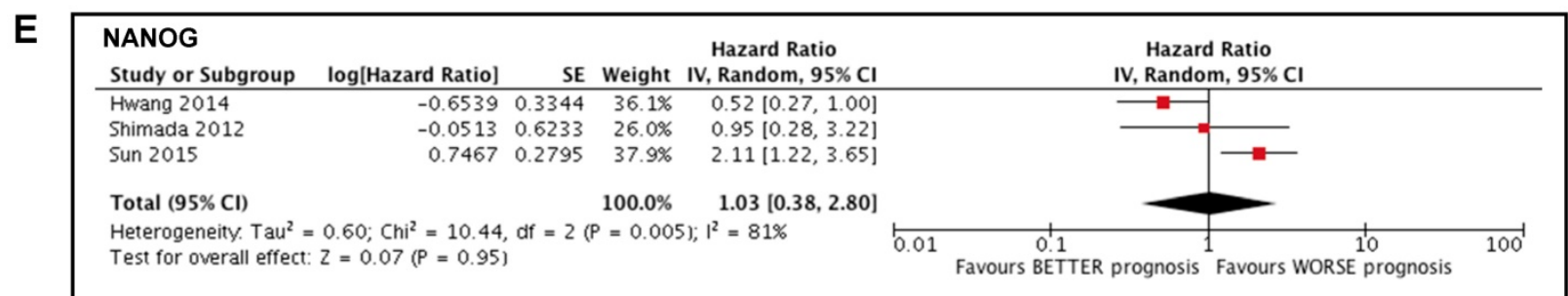

Figure 2. Forest plots of stemness markers associated with OS. (A) CD34 overall survival; (B) CD133 overall survival; (C) Nucleostemin overall survival; (D) OCT-4 overall survival; (E) NANOG overall survival.

\section{Prognostic value of OCT-4 in esophageal cancer}

Four studies [29, 31, 33, 34] examined the expression of OCT -4 and involved 411 patients with SCC that did not undergo neoadjuvant therapy before surgery. Our meta-analysis showed no significant association between OCT-4 expression and the survival of patients affected by esophageal cancer (HR 1.67; 95\% CI 0.88-3.17; I2 82\%; p=0.12) (Figure 2D).

\section{Prognostic value of NANOG in esophageal cancer}

Three studies $[29,39,42]$ involved the analysis of NANOG and included a total of 271 patients with SCC, of which 41 underwent preoperative 
chemoradiation therapy. The meta-analysis that we conducted showed no significant association between the expression of NANOG and the survival of patients affected by esophageal cancer (HR 1.03; 95\% CI 0.38-2.80; I² 81\%; p=0.95) (Figure 2E).

\section{Prognostic value of CD90 in esophageal cancer}

Among the 19 included studies, we did not find any available data about CD90 expression and prognostic value in patients affected by esophageal cancer; therefore, we were not able to conduct a meta-analysis on this marker.

\section{Prognostic value of stem cell markers in patients who did not undergo neoadjuvant therapy}

We conducted a sub-analysis to investigate the potential impact of neoadjuvant treatment on the prognostic value of the stem cells markers, to avoid any bias due to the presence of chemotherapy or radiotherapy. Two studies [41, 43] were excluded from the meta-analysis of CD34 and the sub-analysis showed a significant association between high CD34 expression in tumoral tissue and a poorer prognosis in patients affected by esophageal cancer (HR 2.02; 95\%CI 1.22-3.33; I2 65\%; $\mathrm{p}=0.006$ ) (Figure 3A). Two studies[14, 32] were excluded from the meta-analysis of CD133 and the sub-analysis showed a tendentially significant association between high CD133 expression in tumoral tissue and a poorer prognosis in patients affected by esophageal cancer (HR 1.61; 95\%CI 0.99-2.62; I 59\%; $\mathrm{p}=0.05$ ) (Figure 3B). One study [39] was excluded from the meta-analysis of NANOG and the sub-analysis showed no significant association between the expression of NANOG and the survival of patients affected by esophageal cancer (HR 1.72; 95\%CI 0.87-3.40; I2 27\%; p=0.12) (Figure 3C).

\section{Publication bias}

We performed funnel plot analysis to assess the presence of publication bias in our meta-analysis (Figure 4). The visual inspection of the plots suggests that asymmetry may be present in the graphs of the studies involving CD34 and CD133, whereas studies involving Nucleostemin, OCT-4 and NANOG seem to be free from publication bias. However, we cannot conclude that a significant publication bias is present because real asymmetry is difficult to distinguish from chance when less than 10 studies are included in the analysis [26].
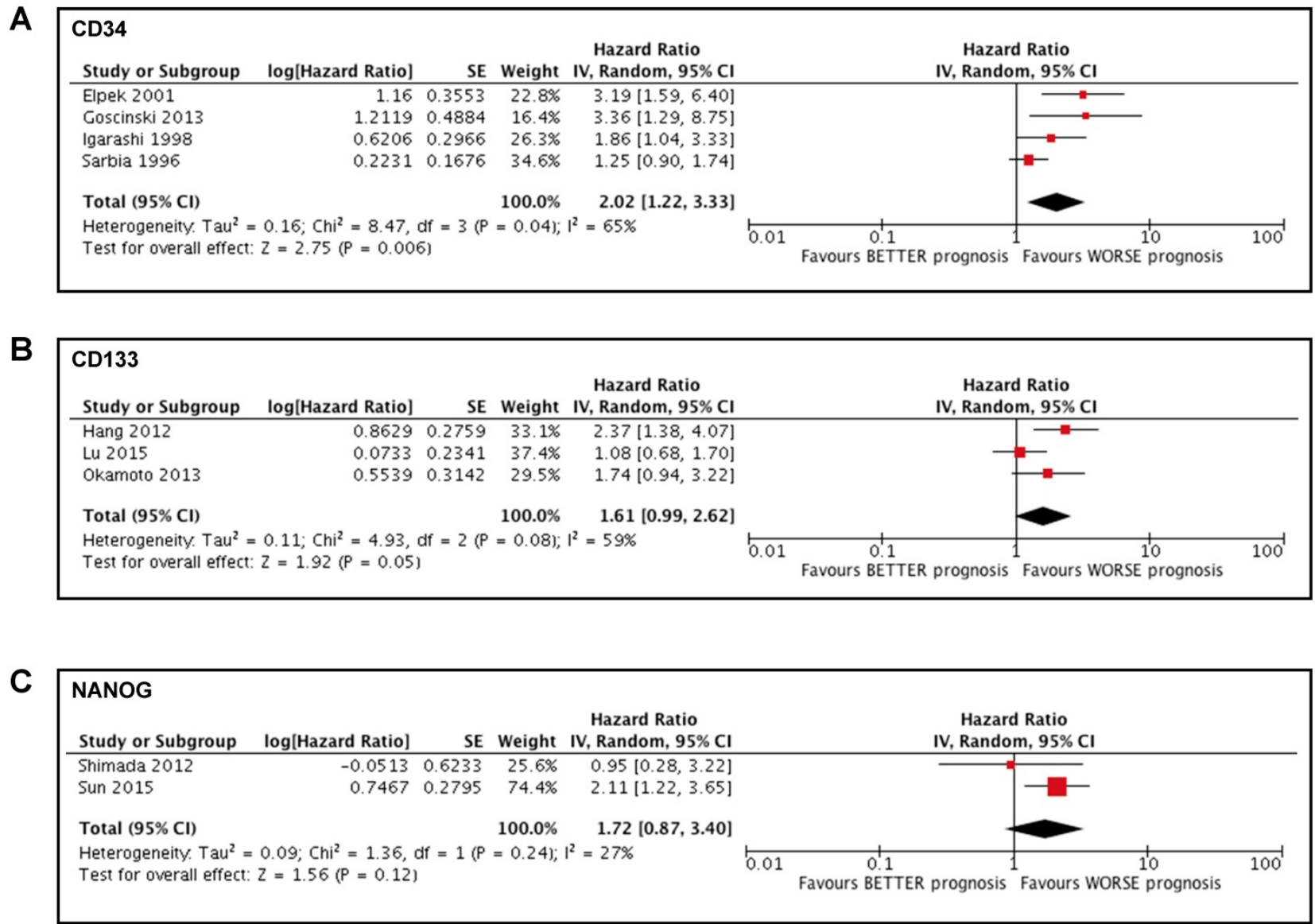

Figure 3. Forest plots of stemness markers in patients who did not undergo neoadjuvant therapy. (A) CD34 overall survival; (B) $C D 133$ overall survival; (C) NANOG overall survival. 
A
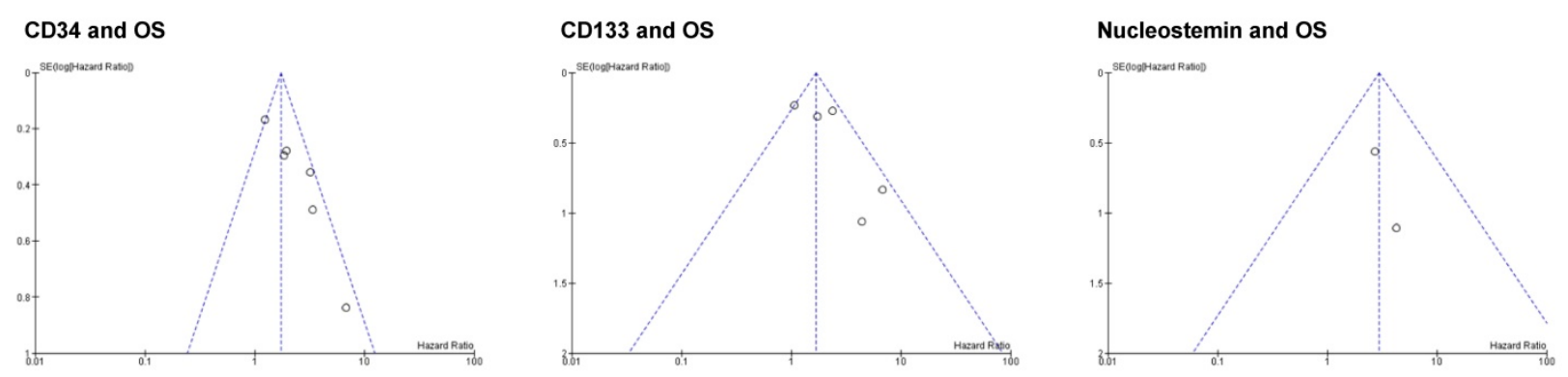

B
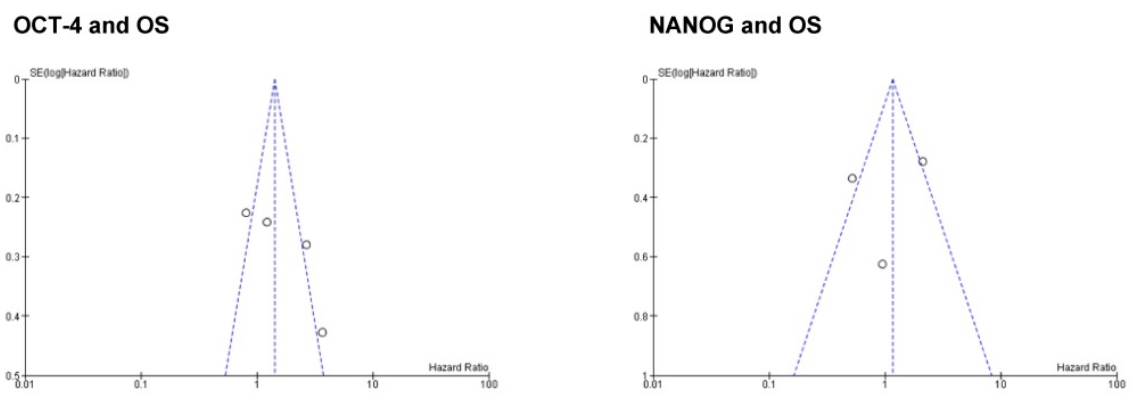

Figure 4. Funnel plots for publication bias test with $\mathbf{9 5 \% c o n f i d e n c e ~ l i m i t s . ~ ( A ) ~ S t e m n e s s ~ m a r k e r s ~ a s s o c i a t e d ~ w i t h ~ O S ; ~ ( B ) ~ S t e m n e s s ~ m a r k e r s ~ n o t ~ a s s o c i a t e d ~ w i t h ~ O S . ~}$

\section{Discussion}

The emerging role of stemness genes as prognostic markers in esophageal cancer needs to be investigated, in order to identify potential prognostic tools that could be more powerful in the management of these malignancies. We identified data from 19 studies that enrolled 1586 patients with esophageal cancer (SCC or EAC). We conducted the meta-analysis for OS and not for DFS because there were less than two studies reporting DFS for each marker. We found that high expression of CD34, CD133 and Nucleostemin in tumor tissue was associated to a poor prognosis in patients affected by esophageal cancer. Data regarding NANOG and OCT-4 showed no significant association between the expression of these markers and OS of patients, whereas no studies involving the measurement of CD90 expression were included in our meta-analysis.

In the studies involving CD34, this stemness marker was measured mostly to assess microvascular density (MVD) in tumoral tissue, to investigate the potential prognostic value of microvascularization in different analyses. With an HR of 2.10 and a 95\%CI from 1.41 to 3.14 , CD34 emerges as the strongest prognostic factors among the markers analyzed in this meta-analysis. This result suggests that high MVD has a prognostic value in esophageal cancer, as recently concluded in another meta-analysis [45]. Tumor angiogenesis is a multi-step process that allows exchange of nutrients, oxygen and growth factors between cancer cells and blood stream, favoring tumor growth and spread [46]. CD34 is expressed by endothelial precursor cells that play a crucial role during angiogenesis, although its function is not fully elucidated [47]. High MVD, measured as CD34 expression, has been shown to have significant prognostic value in different types of cancer: it is associated with poor survival in non-small-cell lung carcinoma (NSCLC) [48] and colorectal cancer [49], and with recurrence in bladder [50] and prostate [51] cancer. It is therefore conceivable that the percentage of cells expressing CD34 may represent a powerful tool to also assess prognosis in esophageal cancer patients.

CD133 is a key factor in epithelial-mesenchimal transition processes, it has been recognized as a marker of cancer stem cells in several type of solid tumors [52] and its biological functions include tumor initiation, cellular migration, vasculogenic mimicry and drug resistance [53]. Although the role of CD133 has not been fully understood, it has been hypothesized that its expression may confer self-renewal capacity, dedifferentiation/stem cell-like properties and anti-apoptotic behavior to a specific population of esophageal cancer stem cells, thus promoting chemoresistance and tumor recurrence [54]. This process may be induced by the inhibition of miR-377, that specifically targets the 3'-UTR binding 
site of CD133, as suggested by a recent study [55] but the underlying mechanism still needs to be clarified.

Nucleostemin upregulation was associated with poor prognosis in hepatocellular carcinoma [56], breast cancer [57], gastric cancer [58] and oral squamous cell carcinoma [15]. In esophageal cells, Nucleostemin may promote cell proliferation via p21 inhibition, as suggested by a study on an esophageal squamous carcinoma cell line [59], but further studies are required to examine more deeply the role of Nucleostemin in tumor progression and/or chemoresistance.

Data involving NANOG and OCT-4 were characterized by elevated heterogeneity $(81 \%$ and $82 \%$ respectively) and we observed a remarkable variability among the cut-off values used in the different studies to determine the positive staining for each marker. In particular, OCT- 4 cut-off values ranged from $>3$ (of a score from 0 to 9 ) to $\geq 2$ (of a score from 0 to 3 ), whereas NANOG cut-off ranged from $>0$ to $>8$ (of a score from 0 to 12 ). This may have led to inconclusive results and more studies are therefore needed to assess the prognostic significance of these two markers.

We conducted a sub-analysis to explore the potential effect of neoadjuvant therapy in the prognostic value of the markers that we examined. Given the impossibility of accurately discriminating patients that received neoadjuvant treatment in each study, we decide to exclude the articles involving chemotherapy and/or radiotherapy from the meta-analyses that included them. The sub-analyses showed that, after the exclusion of all the patients that received neoadjuvant treatment, the prognostic value was significant for CD34 while it tended to be significant for CD133 and remained not significant for NANOG. These results are consistent with the results that included patients who underwent neoadjuvant treatment, suggesting that the presence of preoperative chemotherapy or radiotherapy may not affect the prognostic relevance of these markers on the survival of patients affected by esophageal cancer.

This meta-analysis has some limitations that must be taken into consideration: 15 of the 19 included studies were conducted in Asians and the remaining 4 were conducted in Caucasians, which may produce potential population selection bias. Furthermore, not uniform cut-off values to assess high and low expression of stemness markers may have affected the results of this meta-analysis. Moreover, the selected studies that reported data on EAC [27, 35] considered different outcomes (RFS and CS), making the data unsuitable for a sub-analysis to detect any differences, if present, between EAC and SCC. Despite these limitations, we provided a comprehensive analysis of the association between CD34, CD133, Nucleostemin, OCT-4, NANOG and CD90 stemness markers and OS of patients affected by esophageal cancer. In summary, our meta-analysis revealed that high expression of CD34, CD133, and Nucleostemin was significantly associated with poor OS, suggesting that these stemness markers are promising prognostic factors in patients affected by esophageal cancer [60].

\section{Acknowledgements}

We thank Marco Scarpa for the contribution to the study and Christina A. Drace for the final language editing of the manuscript.

\section{Funding}

This work was supported by Italian Ministry of Health (Ricerca Finalizzata 2016, Prot. RF-201602363566 to R.V.).

\section{Competing Interests}

The authors have declared that no competing interest exists.

\section{References}

1. Domper Arnal MJ, Ferrandez Arenas A, Lanas Arbeloa A. Esophageal cancer: Risk factors, screening and endoscopic treatment in Western and Eastern countries. World J Gastroenterol. 2015; 21: 7933-43.

2. Zhang Y. Epidemiology of esophageal cancer. World J Gastroenterol. 2013; 19: 5598-606.

3. Muller M, Hermann PC, Liebau S, et al. The role of pluripotency factors to drive stemness in gastrointestinal cancer. Stem Cell Res. 2016; 16: 349-57.

4. Issa A, Gill JW, Heideman MR, et al. Combinatorial targeting of FGF and ErbB receptors blocks growth and metastatic spread of breast cancer models. Breast Cancer Res. 2013; 15(R8): 1-15.

5. Miyata Y, Mitsunari K, Asai A, et al. Pathological significance and prognostic role of microvessel density, evaluated using CD31, CD34, and CD105 in prostate cancer patients after radical prostatectomy with neoadjuvant therapy. Prostate. 2015; 75: 84-91.

6. Imdahl A, Bognar G, Schulte-Monting J, et al. Predictive factors for response to neoadjuvant therapy in patients with oesophageal cancer. European journal of cardio-thoracic surgery. 2002; 21: 657-63.

7. Wu H, Qi XW, Yan GN, et al. Is CD133 expression a prognostic biomarker of non-small-cell lung cancer? A systematic review and meta-analysis. PLoS One. 2014; 9: 1-8.

8. Zhou $\mathrm{Q}$, Chen A, Song $\mathrm{H}$, et al. Prognostic value of cancer stem cell marker CD133 in ovarian cancer: a meta-analysis. Int J Clin Exp Med. 2015; 8: 3080-8.

9. Zhong $\mathrm{C}, \mathrm{Wu} \mathrm{JD}$, Fang $\mathrm{MM}$, et al. Clinicopathological significance and prognostic value of the expression of the cancer stem cell marker CD133 in hepatocellular carcinoma: a meta-analysis. Tumour Biol. 2015; 36: 7623-30.

10. Li Z, Yin S, Zhang L, et al. Clinicopathological characteristics and prognostic value of cancer stem cell marker CD133 in breast cancer: a meta-analysis. Onco Targets Ther. 2017; 10: 859-70.

11. Wang K, Xu J, Zhang J, et al. Prognostic role of CD133 expression in colorectal cancer: a meta-analysis. BMC Cancer. 2012; 12(573): 1-6.

12. Guo Z, Li LQ Jiang JH, et al. Cancer stem cell markers correlate with early recurrence and survival in hepatocellular carcinoma. World J Gastroenterol. 2014; 20: 2098-106.

13. Fiegel HC, Kaifi JT, Quaas A, et al. Lack of Thy1 (CD90) expression in neuroblastomas is correlated with impaired survival. Pediatr Surg Int. 2008; 24: 101-5.

14. Nakajima TE, Yoshida H, Okamoto $\mathrm{N}$, et al. Nucleostemin and TWIST as predictive markers for recurrence after neoadjuvant chemotherapy for esophageal carcinoma. Cancer science. 2012; 103: 233-8.

15. Yoshida R, Nakayama $H$, Nagata $M$, et al. Overexpression of nucleostemin contributes to an advanced malignant phenotype and a poor prognosis in oral squamous cell carcinoma. Br J Cancer. 2014; 111: 2308-15.

16. Tin AS, Park AH, Sundar SN, et al. Essential role of the cancer stem/progenitor cell marker nucleostemin for indole-3-carbinol anti-proliferative responsiveness in human breast cancer cells. BMC Biol. 2014; 12(72): 1-22. 
17. Wen $\mathrm{K}, \mathrm{Fu} \mathrm{Z}, \mathrm{Wu} \mathrm{X}$, et al. Oct-4 is required for an antiapoptotic behavior of chemoresistant colorectal cancer cells enriched for cancer stem cells: effects associated with STAT3/Survivin. Cancer Lett. 2013; 333: 56-65.

18. Achuthan S, Santhoshkumar TR, Prabhakar J, et al. Drug-induced senescence generates chemoresistant stemlike cells with low reactive oxygen species. J Biol Chem. 2011; 286: 37813-29.

19. Rodrigues M, Xavier FCA, Andrade NP, et al. Prognostic implications of CD44, NANOG, OCT4, and BMI1 expression in tongue squamous cell carcinoma. Head Neck. 2018; 40(8):1759-73

20. Lee M, Nam EJ, Kim SW, et al. Prognostic impact of the cancer stem cell-related marker NANOG in ovarian serous carcinoma. Int J Gynecol Cancer. 2012; 22: 1489-96.

21. Nagata T, Shimada Y, Sekine S, et al. KLF4 and NANOG are prognostic biomarkers for triple-negative breast cancer. Breast Cancer. 2017; 24: 326-35.

22. Raufi AG, Klempner SJ. Immunotherapy for advanced gastric and esophageal cancer: preclinical rationale and ongoing clinical investigations. J Gastrointest Oncol. 2015; 6: 561-9.

23. Ajani JA, Wang X, Song S, et al. ALDH-1 expression levels predict response or resistance to preoperative chemoradiation in resectable esophageal cancer patients. Mol Oncol. 2014; 8: 142-9.

24. Bao $\mathrm{S}, \mathrm{Wu} \mathrm{Q}, \mathrm{McLendon} \mathrm{RE}$, et al. Glioma stem cells promote radioresistance by preferential activation of the DNA damage response. Nature. 2006; 444: 756-60.

25. Parmar $\mathrm{MK}$, Torri V, Stewart L. Extracting summary statistics to perform meta-analyses of the published literature for survival endpoints. Stat Med. 1998; 17: 2815-34

26. Higgins JPT, Green S, Cochrane Collaboration. Cochrane handbook for systematic reviews of interventions. Chichester, England; Hoboken, NJ: Wiley-Blackwell; 2008

27. Perry C, Soomro I, Kaye P, et al. Analysis of lymphatic and blood vessel invasion biomarkers in T1 esophagogastric adenocarcinomas for improved patient prognostication. Diseases of the esophagus 2015; 28: 262-8.

28. $\mathrm{Lu} \mathrm{CL}, \mathrm{Xu} \mathrm{FK}, \mathrm{Gu} \mathrm{J}$, et al. Clinical and biological significance of stem-like CD133(+)CXCR4(+) cells in esophageal squamous cell carcinoma. Journal of Thoracic and Cardiovascular Surgery. 2015; 150: 386-95.

29. Shimada Y, Okumura T, Sekine S, et al. Expression analysis of iPS cell inductive genes in esophageal squamous cell carcinoma by tissue microarray. Anticancer research. 2012; 32: 5507-14

30. Zhang G, Zhang Q, Zhang Q, et al. Expression of nucleostemin, epidermal growth factor and epidermal growth factor receptor in human esophageal squamous cell carcinoma tissues. Journal of cancer research and clinical oncology. 2010; 136: 587-94.

31. He W, Li K, Wang F, et al. Expression of OCT4 in human esophageal squamous cell carcinoma is significantly associated with poorer prognosis. World journal of gastroenterology. 2012; 18: 712-9.

32. Okamoto K, Ninomiya I, Ohbatake Y, et al. Expression status of CD44 and CD133 as a prognostic marker in esophageal squamous cell carcinoma treated with neoadjuvant chemotherapy followed by radical esophagectomy. Oncology reports. 2016; 36: 3333-42.

33. Yang Z, Cui Y, Ni W, et al. Gli1, a potential regulator of esophageal cancer stem cell, is identified as an independent adverse prognostic factor in esophageal squamous cell carcinoma. Journal of cancer research and clinical oncology. 2017; 143: 243-54

34. Li C, Yan Y, Ji W, et al. OCT4 positively regulates Survivin expression to promote cancer cell proliferation and leads to poor prognosis in esophageal squamous cell carcinoma. PloS one. 2012; 7(11) e49693: 1-10.

35. Goscinski MA, Nesland JM, Giercksky KE, et al. Primary tumor vascularity in esophagus cancer. CD34 and HIF1-alpha expression correlate with tumor progression. Histology and histopathology. 2013; 28: 1361-8.

36. Elpek GO, Gelen T, Aksoy NH, et al. The prognostic relevance of angiogenesis and mast cells in squamous cell carcinoma of the oesophagus. Journal of clinical pathology. 2001; 54: 940-4.

37. Igarashi $\mathrm{M}$, Dhar DK, Kubota $\mathrm{H}$, et al. The prognostic significance of microvessel density and thymidine phosphorylase expression in squamous cell carcinoma of the esophagus. Cancer. 1998; 82: 1225-32.

38. Hang D, Dong HC, Ning T, et al. Prognostic value of the stem cell markers CD133 and ABCG2 expression in esophageal squamous cell carcinoma. Diseases of the esophagus. 2012; 25: 638-44.

39. Hwang $\mathrm{CC}$, Nieh $\mathrm{S}$, Lai $\mathrm{CH}$, et al. A retrospective review of the prognostic value of ALDH-1, Bmi-1 and Nanog stem cell markers in esophageal squamous cell carcinoma. PloS one. 2014; 9(8) e105676: 1-7.

40. Okamoto H, Fujishima F, Nakamura Y, et al. Significance of CD133 expression in esophageal squamous cell carcinoma. World journal of surgical oncology. 2013; 11 (51): 1-7.

41. Kitadai Y, Haruma K, Tokutomi T, et al. Significance of vessel count and vascular endothelial growth factor in human esophageal carcinomas. Clinical Cancer Research. 1998; 4: 2195-200.

42. Sun LL, Wu JY, Wu ZY, et al. A three-gene signature and clinical outcome in esophageal squamous cell carcinoma. International journal of cancer. 2015; 136: 569-77.

43. Tanigawa N, Matsumura M, Amaya $H$, et al. Tumor vascularity correlates with the prognosis of patients with esophageal squamous cell carcinoma. Cancer. 1997; 79: 220-5.
44. Sarbia $\mathrm{M}$, Bittinger $\mathrm{F}$, Porschen $\mathrm{R}$, et al. Tumor vascularization and prognosis in squamous cell carcinomas of the esophagus. Anticancer research. 1996; 16: 2117-21.

45. Ma G, Zhang J, Jiang H, et al. Microvessel density as a prognostic factor in esophageal squamous cell cancer patients: A meta-analysis. Medicine (Baltimore). 2017; 96(29) e7600: 1-6.

46. Bettencourt MC, Bauer JJ, Sesterhenn IA, et al. CD34 immunohistochemical assessment of angiogenesis as a prognostic marker for prostate cancer recurrence after radical prostatectomy. J Urol. 1998; 160: 459-65.

47. Sidney LE, Branch MJ, Dunphy SE, et al. Concise review: evidence for CD34 as a common marker for diverse progenitors. Stem Cells. 2014; 32: 1380-9.

48. Mineo TC, Ambrogi V, Baldi A, et al. Prognostic impact of VEGF, CD31, CD34, and CD105 expression and tumour vessel invasion after radical surgery for IB-IIA non-small cell lung cancer. Journal of clinical pathology. 2004; 57: 591-7.

49. Ma YL, Peng JY, Zhang P, et al. Immunohistochemical analysis revealed CD34 and Ki67 protein expression as significant prognostic factors in colorectal cancer. Med Oncol. 2010; 27: 304-9.

50. Ajili F, Kacem M, Tounsi H, et al. Prognostic impact of angiogenesis in nonmuscle invasive bladder cancer as defined by microvessel density after immunohistochemical staining for CD34. Ultrastruct Pathol. 2012; 36: 336-42.

51. Nassif AE, Tambara Filho R. Immunohistochemistry expression of tumor markers CD34 and P27 as a prognostic factor of clinically localized prostate adenocarcinoma after radical prostatectomy. Rev Col Bras Cir. 2010; 37: $338-44$

52. Grosse-Gehling P, Fargeas CA, Dittfeld C, et al. CD133 as a biomarker for putative cancer stem cells in solid tumours: limitations, problems and challenges. J Pathol. 2013; 229: 355-78.

53. Nadal R, Ortega FG, Salido M, et al. CD133 expression in circulating tumor cells from breast cancer patients: potential role in resistance to chemotherapy. International journal of cancer. 2013; 133: 2398-407.

54. Sui YP, Jian XP, Ma LI, et al. Prognostic value of cancer stem cell marker CD133 expression in esophageal carcinoma: A meta-analysis. Molecular and clinical oncology. 2016; 4: 77-82

55. Li B, Xu WW, Han L, et al. MicroRNA-377 suppresses initiation and progression of esophageal cancer by inhibiting CD133 and VEGF. Oncogene. 2017; 36: 3986-4000.

56. Hua L, Hu B, Yan D, et al. Upregulated expression of Nucleostemin/GNL3 is associated with poor prognosis and Sorafenib Resistance in Hepatocellular Carcinoma. Pathol Res Pract. 2017; 213: 688-97.

57. Kobayashi T, Masutomi K, Tamura K, et al. Nucleostemin expression in invasive breast cancer. BMC Cancer. 2014; 14(215): 1-9.

58. Wu $\mathrm{H}$, Wang $\mathrm{W}$, Tong $\mathrm{S}$, et al. Nucleostemin regulates proliferation and migration of gastric cancer and correlates with its malignancy. Int J Clin Exp Med. 2015; 8: 17634-43.

59. Sun $Y$, Tan $X$, Tang $Z$, et al. Knockdown of nucleostemin can inhibit the proliferation of esophageal carcinoma cells in vitro through upregulating p21. Hepato-gastroenterology. 2014; 61: 2247-52.

60. Carraro A, Trevellin E, Fassan $\mathrm{M}$, et al. Esophageal adenocarcinoma microenvironment: Peritumoral adipose tissue effects associated with chemoresistance. Cancer science. 2017; 108(12): 2393-404. 